\title{
Dual-mode photoacoustic microscopy of carbon nanotube incorporated scaffolds in blood and biological tissues
}

Xin Cai, Song Hu, Bhavna Paratala, Balaji Sitharaman, Lihong V. Wang

Xin Cai, Song Hu, Bhavna Paratala, Balaji Sitharaman, Lihong V. Wang, "Dual-mode photoacoustic microscopy of carbon nanotube incorporated scaffolds in blood and biological tissues," Proc. SPIE 7899, Photons Plus Ultrasound: Imaging and Sensing 2011, 78992I (18 February 2011); doi: $10.1117 / 12.873230$

SPIE. Event: SPIE BiOS, 2011, San Francisco, California, United States 


\title{
Dual-mode Photoacoustic Microscopy of Carbon Nanotube Incorporated Scaffolds in Blood and Biological Tissues
}

\author{
Xin $\mathrm{Cai}^{1}$, Song $\mathrm{Hu}^{1}$, Bhavna Paratala ${ }^{2}$, Balaji Sitharaman ${ }^{2}$, and Lihong V. Wang ${ }^{1 *}$ \\ ${ }^{1}$ Optical Imaging Laboratory, Department of Biomedical Engineering, Washington University in St. \\ Louis, St. Louis, Missouri 63130 \\ ${ }^{2}$ Department of Biomedical Engineering, State University of New York at Stony Brook, Stony \\ Brook, NewYork 11794
}

\begin{abstract}
Three-dimensional scaffolds provide physical support and an adjustable microenvironment to facilitate vascularization of ischemic tissues; however, in vivo imaging of scaffold functioning is still challenging. Micro-CT, the current frequentlyused imaging modality for scaffold characterization, provides poor contrast for wet scaffold, which limits its in vivo applications. In this paper, dual modes of photoacoustic microscopy (PAM), using acoustic resolution PAM (AR-PAM) and optical resolution PAM (OR-PAM), were employed for imaging scaffolds in blood as well as in chicken breast tissues. By choosing different wavelengths, $570 \mathrm{~nm}$ and $638 \mathrm{~nm}$, we spectroscopically differentiated the photoacoustic signals generated from blood and from carbon nanotube incorporated scaffolds. The ex vivo experiments demonstrated a lateral resolution of $45 \mu \mathrm{m}$ and a maximum penetration of $\sim 2 \mathrm{~mm}$ for AR-PAM, and a lateral resolution of $3 \mu \mathrm{m}$ and a maximum penetration of $\sim 660 \mu \mathrm{m}$ for OR-PAM. OR-PAM further quantified the average pore size of scaffolds to be $100-200 \mu \mathrm{m}$ in diameter. Our results suggest that PAM is a promising tool for in vivo monitoring of scaffold-induced angiogenesis as well as the degradability of scaffolds themselves.
\end{abstract}

Keywords: Three-dimensional scaffold; photoacoustic microscopy; carbon nanotube.

\section{INTRODUCTION}

Tissue engineering is promising for tissue defect repair. Three-dimensional (3D) porous polymer scaffolds can provide physical supports for angiogenesis, which is one of the most important steps in the process of tissue repair and wound healing. ${ }^{1}$ The scaffolds must have good properties for implantation, such as biocompatibility, biodegradability, mechanical strength, porosity, pore size, interconnectivity, and so on. Non-invasive in vivo imaging of scaffolds is critical for assessing the properties of scaffolds and the vascularization process of the regenerating tissue. ${ }^{2}$ However, a significant difficulty in scaffold imaging is the lack of a high-resolution imaging technique that can penetrate deeply and nondestructively into the scaffold. For example, optical microscopy, including confocal and two-photon laser scanning microscopy, has high resolution, but it is typically limited to several hundred micrometers penetration due to strong light scattering. Micro-computed tomography (micro-CT) based on X-ray can penetrate a whole construct up to several centimeters. However, it provides poor contrast for wet scaffolds, which limits its in vivo applications. So, there is still a strong need for a non-invasive imaging modality with high resolution and deep penetration for 3D scaffolds.

The emerging photoacoustic microscopy (PAM) is attractive for imaging scaffolds in a non-invasive manner. Recently, Zhang et al reported PAM imaging of melanoma cell distribution in scaffolds for tissue engineering applications. ${ }^{3}$ PAM detects photoacoustic waves generated from the objects that absorb either pulsed or intensity-modulated laser

*Corresponding author: lhwang@biomed.wustl.edu

Photons Plus Ultrasound: Imaging and Sensing 2011, edited by Alexander A. Oraevsky, Lihong V. Wang, Proc. of SPIE Vol. 7899, 78992I - (C) 2011 SPIE · CCC code: 1605-7422/11/\$18 - doi: 10.1117/12.873230 
irradiation. ${ }^{4}$ The non-ionizing irradiation in PAM imaging imposes no hazardous effects to tissues, in contrast with ionizing X-rays in micro-CT. Additionally, PAM is capable of multi-scale imaging, bridging the resolution and penetration gaps between the tissue/organ level and the cellular level. ${ }^{5}$ With weakly focused optical illumination, millimeter penetration with a tissue level resolution has been demonstrated by acoustic-resolution photoacoustic microscopy (AR-PAM). ${ }^{6}$ Utilizing diffraction-limited optical focusing, optical-resolution photoacoustic microscopy (OR-PAM), has achieved cellular resolution in vivo ${ }^{7}$. PAM enables in vivo label-free vascular imaging with high sensitivity, because hemoglobin, the primary carrier of oxygen in the blood, has a strong intrinsic contrast for PAM in the range of visible light. This unique feature allows PAM to avoid possible alterations to the intrinsic hemodynamics caused by exogenous angiographic agents. ${ }^{8}$

Here, we report both AR-PAM and OR-PAM for non-invasive imaging of single-walled carbon nanotube (SWCNT)incorporated poly(D, L-lactide-co-glycolide) (PLGA) scaffolds in blood and tissue phantoms. By choosing different wavelengths, $570 \mathrm{~nm}$ and $638 \mathrm{~nm}$, we spectroscopically differentiated the photoacoustic signals generated from blood and from SWCNT incorporated scaffolds, which can be potentially used for in vivo monitoring the process of scaffoldinduced angiogenesis and scaffold degradability simultaneously.

\section{MATERIALS AND METHODS}

\subsection{SWCNTs synthesis}

The method of diblock copolymer templating was used to coat Fe coated on Si wafers. The wafers were placed in a 3-in. quartz reaction chamber (Easy Tube 2000, First Nano) and heated in Ar to $900{ }^{\circ} \mathrm{C}$. To initiate the growth of SWCNTs, the chamber was filled with $\mathrm{H}_{2}$ for $2 \mathrm{~min}$, and $\mathrm{CH}_{4}$ was added to the gas flow as the carbon feedstock for $20 \mathrm{~min}$. Subsequently, the carbon feedstock was switched off and the furnace was cooled to room temperature. ${ }^{9}$

\subsection{Preparation of SWCNTs-incorporated PLGA scaffolds}

Porous SWCNT-incorporated PLGA (50:50 lactic-to glycolic-acid copolymer; Sigma Aldrich) scaffolds (4 mm x 1.5 $\mathrm{mm}$ ) were prepared using an established procedure of high shear mixing, sonicating and drying. ${ }^{10}$ SWCNTs were uniformly distributed into the PLGA mixture at $0.5 \mathrm{wt} \%$ concentration, because the composites achieved their maximum mechanical properties at this loading concentration. ${ }^{11}$ Porous scaffolds were fabricated by a thermal-crosslinking particulate-leaching technique with $\mathrm{NaCl}$ porogen (size range: $100-500 \mu \mathrm{m}) .{ }^{10}$

\subsection{Photoacoustic microscopy}

Figure 1 shows the schematic of the systems. For photoacoustic excitation, a dye laser (CBR-D, Sirah, Kaarst, Germany) pumped by a Nd:YLF laser (INNOSLAB, Edgewave, Wuerselen, Germany) was employed to provide 7-ns laser pulses with a repetition rate up to $5 \mathrm{kHz}$. The optical and ultrasonic foci are configured coaxially and confocally. The lateral resolution is dominated by the more tightly focused part. The axial resolution and the maximum penetration depth are inversely proportional to the center frequency of the transducer. ${ }^{12}$

For AR-PAM, The optical focus is $2 \mathrm{~mm}$ in diameter, which is much wider than the $45-\mu \mathrm{m}$ ultrasonic focus. ${ }^{7}{ }^{13}$ Using a focused ultrasonic transducer with $50 \mathrm{MHz}$ central frequency (V214-BB-RM, Olympus NDT, Kennewick, WA), ARPAM could achieve $45 \mu \mathrm{m}$ lateral resolution, $15 \mu \mathrm{m}$ axial resolution, and more than $3 \mathrm{~mm}$ penetration depth. For ORPAM, a diffraction-limited bright-field optical illumination, which is much smaller in diameter than the acoustic focus, is adopted to achieve optical resolution. The lateral resolution of OR-PAM was $3 \mu \mathrm{m}$, which is capable of resolving microvasculature as small as single capillaries. However, the tissue penetration of OR-PAM is restricted to $\sim 1 \mathrm{~mm}$ due to the high optical scattering. ${ }^{14}$ 

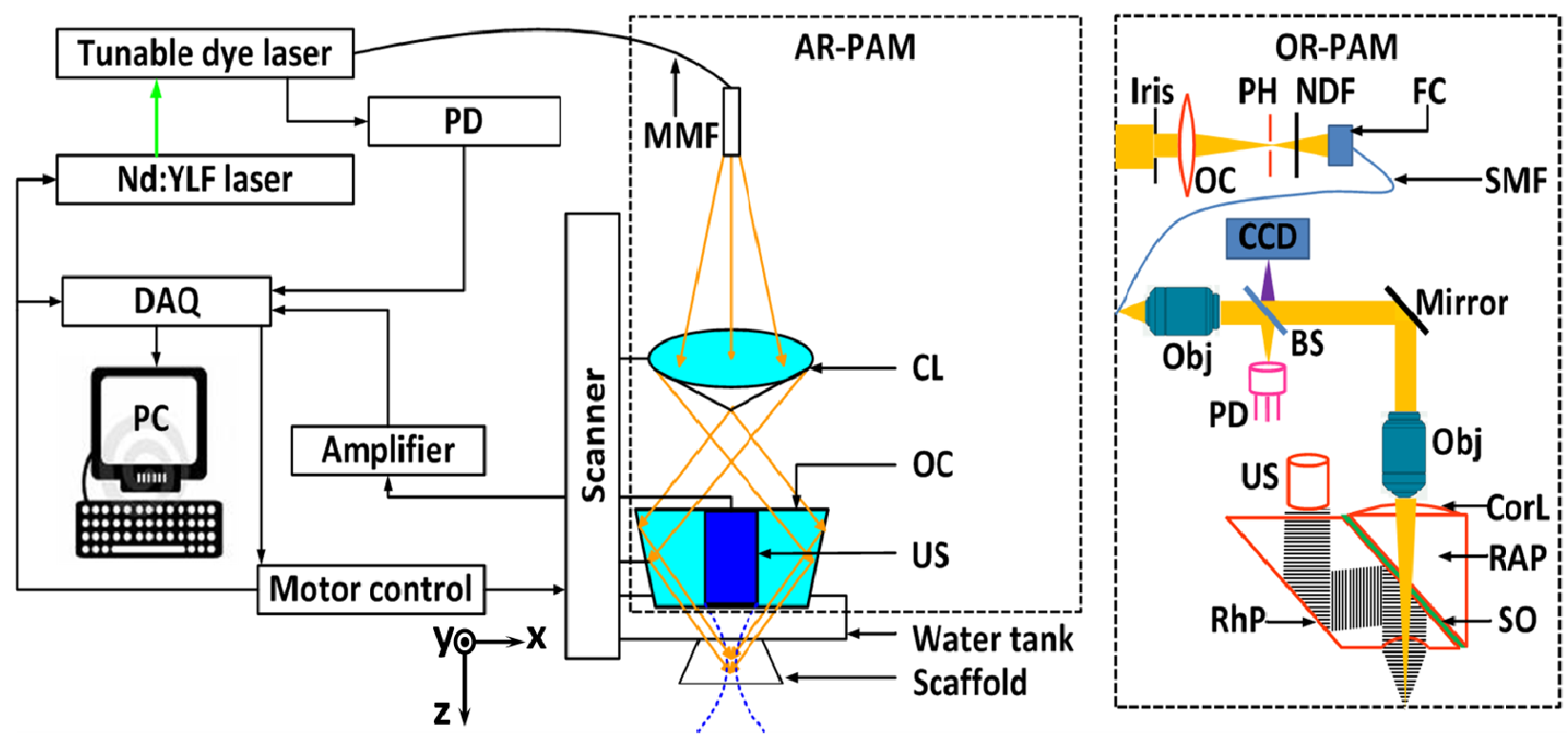

Fig. 1. Schematic of the reflection-mode AR-PAM and OR-PAM systems. A Cartesian coordinate is also shown in the drawing. BS: Beam splitter; CCD: charge-coupled device; CL: Conical lens; CorL: Correction lens; FC: Fiber collimator; MMF: Multi-mode fiber; NDF: Neutral density filter; Obj: Objective; OC: Optical condenser; PD: Photodiode; PH: Pinhole; RAP: Right-angle prism; RhP: Rhomboid prism; SMF: Single-mode fiber; SO: Silicone oil; US: Ultrasonic transducer.

\section{RESULTS}

The scaffolds, immersing in bovine blood (905-250, Quadfive) and chicken tissue, were imaged by AR-PAM and ORPAM systems. Figure 2 shows PAM x-y projection (Figure 2a-2d) and $x-z$ projection (Figure 2e-2h) images of the SWCNT- incorporated scaffold in the bovine blood at different wavelengths. At the $570 \mathrm{~nm}$ wavelength, the blood signal is dominant and masks the signal from the scaffold. In contrast, at the $638 \mathrm{~nm}$ wavelength, only the SWCNTincorporated scaffold gives strong photoacoustic signal. In Figure 2d, OR-PAM shows more concise and clear details of the scaffold in $\mathrm{x}-\mathrm{y}$ projection image due to its high lateral resolution, and we can further quantify that the average pore size of the scaffold is 100-200 $\mu \mathrm{m}$ in diameter. In Figure 2f, AR-PAM shows a capability of imaging the scaffold at $\sim 2$ $\mathrm{mm}$ depth into the blood. This penetration depth is rather deep as compared to the conventional microscopy techniques. AR-PAM system could still provide acceptable resolution at such a deep penetration depth, because the resolution is determined by the ultrasound parameters and ultrasound scattering is much weaker than optical scattering in biological tissues.

Figure 3 shows PAM x-y projection (Figure 3a-3d) and $x-z$ projection (Figure 3e-3h) images of the SWCNTincorporated scaffold in the bovine blood at different wavelengths. The scaffold is seen and the photoacoustic signal is similar at both wavelengths, which indicates that the tissue absorption is weak at these wavelengths. As shown in Figure 3e-3f, AR-PAM still can penetrate the tissue-covered scaffold more than $1 \mathrm{~mm}$. In contrast, OR-PAM is defocused by the tissue and only images a small part of the scaffold in shallower region. 


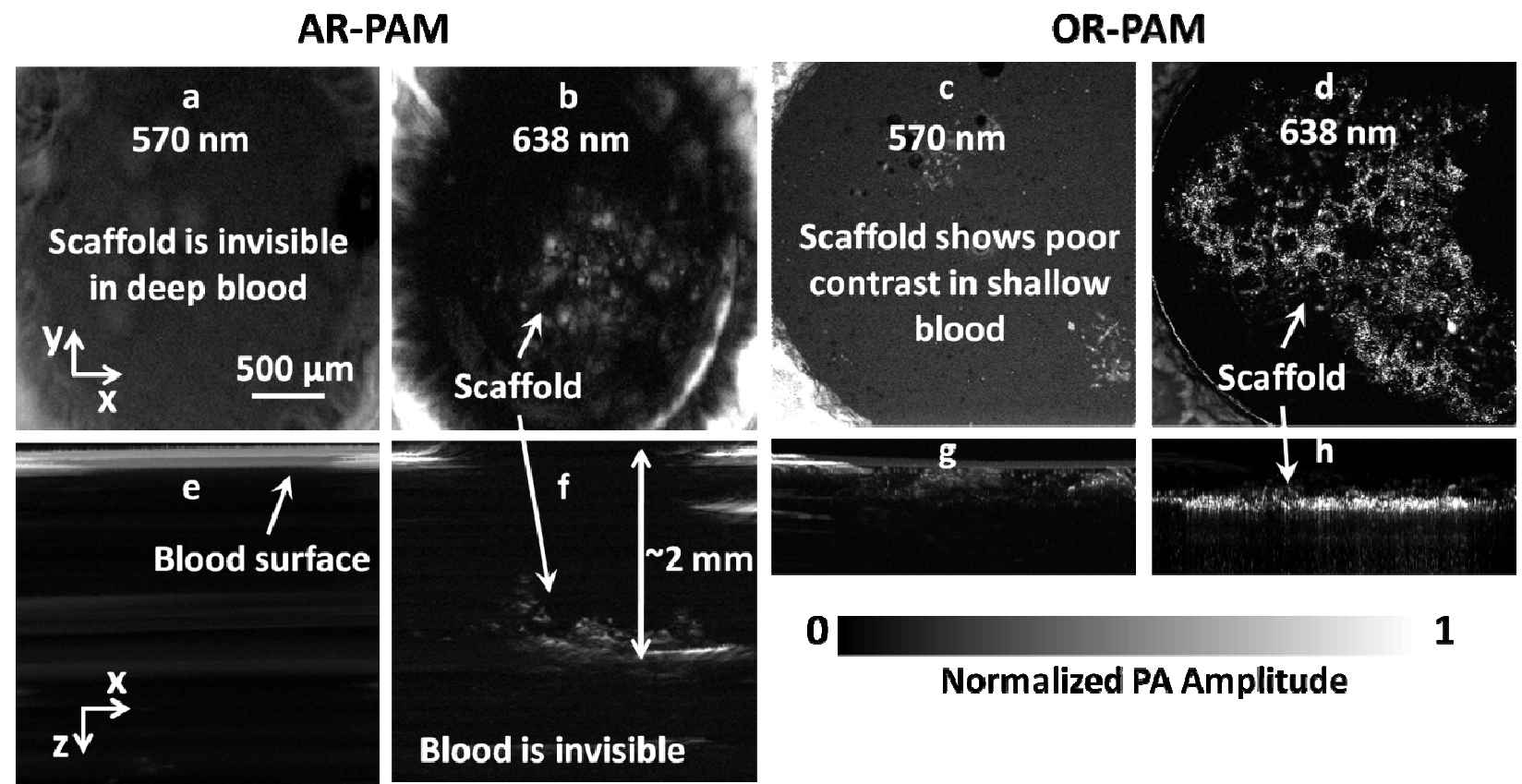

Fig. 2. PAM x-y projection and x-z projection images of the SWCNT-incorporated scaffold in bovine blood at different wavelengths (a) AR-PAM x-y projection image at 570nm. (b) AR-PAM x-y projection image at $638 \mathrm{~nm}$. (c) OR-PAM x-y projection image at $570 \mathrm{~nm}$. (d) OR-PAM x-y projection image at $638 \mathrm{~nm}$. (e) Corresponding $\mathrm{x}-\mathrm{z}$ projection image for (a). (f) Corresponding $\mathrm{x}-\mathrm{z}$ projection image for (b), which shows the scaffold is $\sim 2 \mathrm{~mm}$ under the blood. (g) Corresponding $\mathrm{x}-\mathrm{z}$ projection image for (c). (h) Corresponding $\mathrm{x}-\mathrm{z}$ projection image for $(\mathrm{d})$.
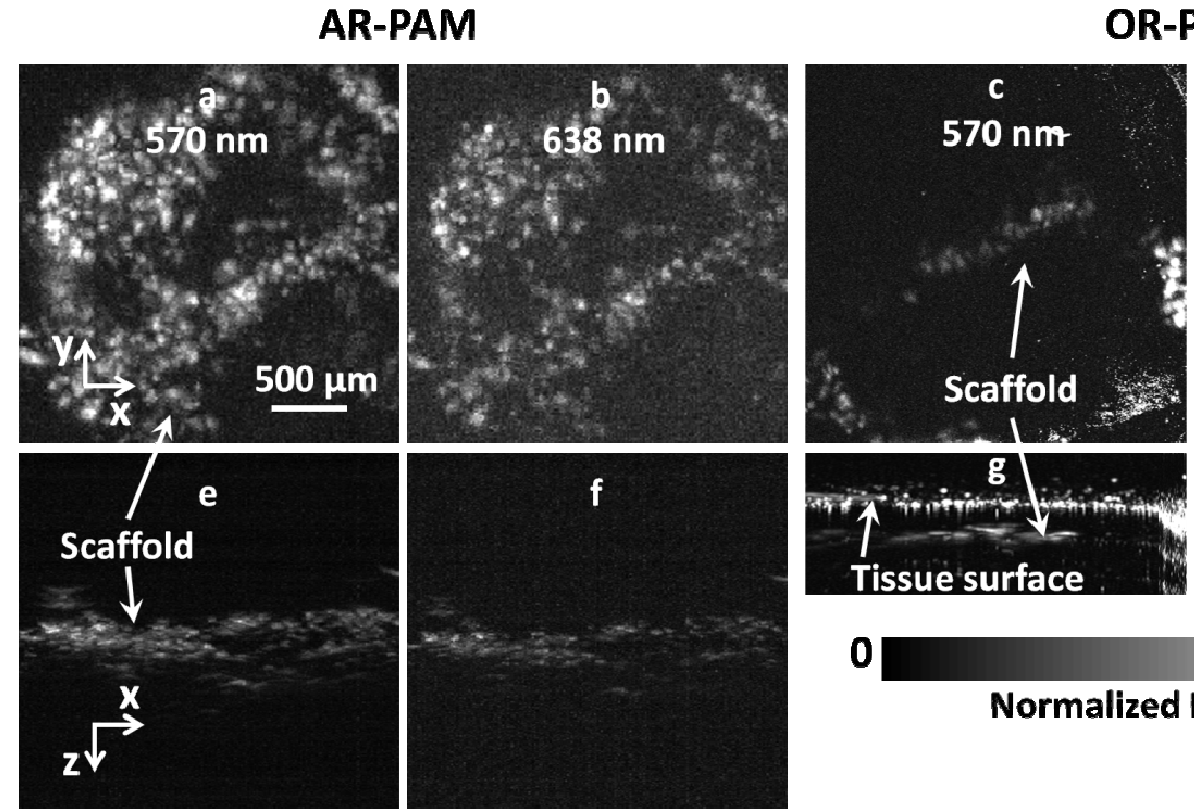

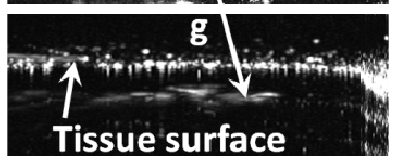

0

\section{Normalized PA Amplitude}

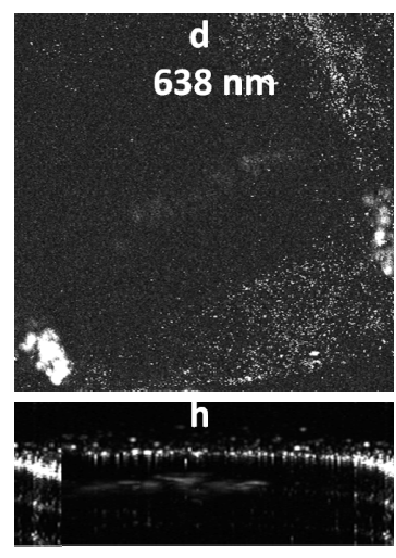

1

3. PAM $x-y$ projection and $x-z$ projection images of the SWCNT-incorporated scaffold in chicken breast tissue at different wavelengths. (a) AR-PAM x-y projection image at 570nm. (b) AR-PAM x-y projection image at 638nm. (c) OR-PAM x-y projection image at $570 \mathrm{~nm}$. (d) OR-PAM $x-y$ projection image at $638 \mathrm{~nm}$. (e) Corresponding $x-z$ projection image for (a). (f) Corresponding $x-z$ projection image for (b). (g) Corresponding x-z projection image for (c). (h) Corresponding x-z projection image for (d). 


\section{CONCLUSION}

We have demonstrated that dual-mode PAM can be used as an imaging tool for SWCNT incorporated scaffolds in blood and biological tissue. OR-PAM has better lateral resolution than AR-PAM. It is good for ex vivo quantification of the scaffolds. Pore size of the scaffolds estimated by OR-PAM is 100-200 $\mu \mathrm{m}$ in diameter. However, AR-PAM has a larger penetration: 1) In chicken tissue, OR-PAM is defocused and only imaged small part of the scaffold locating at the superficial; AR-PAM is affected little because of its dark-field illumination. 2) AR-PAM can penetrate the whole scaffold; OR-PAM can only image the top layer of the scaffold. Thus, for future in vivo experiments, AR-PAM might be a better tool. Our results suggest that PAM is a promising tool for in vivo monitoring of scaffold-induced angiogenesis as well as the degradability of scaffolds themselves.

\section{ACKNOWLEDGMENTS}

This work was sponsored by National Institutes of Health grants No. 1DP2OD007394-01(to B.S.), No. R01 EB000712, No. R01 EB008085, No. R01 CA134539, and No. U54 CA136398 (Network for Translational Research) (to L.V.W.). L.V.W. has a financial interest in Microphotoacoustics, Inc. and Endra, Inc., which, however, did not support this work.

\section{REFERENCES}

[1] R. Langer, J. P. Vacanti, “Tissue engineering,” Science 260, 920-26 (1993).

[2] M. Lovett, K. Lee, A. Edwards, D. L. Kaplan, "Vascularization Strategies for Tissue Engineering," Tissue Eng Part B: Reviews 15 (3), 353-370 (2009).

[3] Y. Zhang, X. Cai, S.-W. Choi, C. Kim, L. V. Wang, Y. Xia, "Chronic label-free volumetric photoacoustic microscopy of melanoma cells in three-dimensional porous Scaffolds," Biomaterials 31, 8651-8658 (2010).

[4] C. Kim, C. Favazza, L. V. Wang, "In vivo photoacoustic tomography of chemicals: high-resolution functional and molecular optical imaging at new depths," Chem Rev 110, 2756-82 (2010).

[5] L. V. Wang, "Multiscale photoacoustic microscopy and computed tomography," Nature Photon 3(9), 503-509 (2009).

[6] H. F. Zhang, K. Maslov, G. Stoica, L. V. Wang, "Functional photoacoustic microscopy for high-resolution and noninvasive in vivo imaging," Nat Biotechnol 24, 848-51 (2006).

[7] K. Maslov, H. F. Zhang, S. Hu, L. V. Wang, "Optical-resolution photoacoustic microscopy for in vivo imaging of single capillaries," Opt Lett 33, 929-931 (2008).

[8] S. Hu, L. V. Wang, "Photoacoustic imaging and characterization of the microvasculature," J Biomed Opt 15(1), 011101 (2010).

[9] Q. Fu, S. M. Huang, J. Liu, "Chemical vapor depositions of single-walled carbon nanotubes catalyzed by uniform $\mathrm{Fe}_{2} \mathrm{O}_{3}$ nanoclusters synthesized using diblock copolymer micelles," J. Phys. Chem. B 108(20), 6124-6129 (2004).

[10] X. Shi, B. Sitharaman, Q. P. Pham, K. Wu, W. Edward Billups, L. J. Wilson, A. G. Mikos, "Fabrication of Porous Ultra-Short Single-Walled Carbon Nanotube Nanocomposite Scaffolds for Bone Tissue Engineering," Biomaterials 28, 4078-4090 (2007).

[11] B. Sitharaman, X. Shi, L. A. Tran, P. P. Spicer, I. Rusakova, L. J. Wilson, A. G. Mikos, "Injectable in situ crosslinkable nanocomposites of biodegradable polymers and carbon nanostructures for bone tissue engineering," $J$ Biomater Sci Polym Ed 18(6), 655-71 (2007).

[12] S. Hu, L. V. Wang, "Neurovascular photoacoustic tomography," Front Neuroenergetics 2(10), doi:10.3389/fnene.2010.00010 (2010) 
[13] K. Maslov, G. Stoica, L. V. Wang, "In vivo dark-field reflection-mode photoacoustic microscopy," Opt Lett 30, 625-7 (2005).

[14] S. Hu, K. Maslov, L. V. Wang, "In vivo functional chronic imaging of a small animal model using optical-resolution photoacoustic microscopy,” Med Phys, 36(6), 2320-2323 (2009). 